University of Nebraska - Lincoln

DigitalCommons@University of Nebraska - Lincoln

$1-2018$

\title{
Alcohol Intoxication Moderates the Association between a Polygenic Risk Score and Unprovoked Intimate Partner Aggression
}

\author{
Christa C. Christ \\ Hamilton College, cchrist@uscupstate.edu \\ Laura E. Watkins \\ Yale School of Medicine, laura.e.watkins@emory.edu \\ David DiLillo \\ University of Nebraska-Lincoln, ddilillo@unl.edu \\ Scott F. Stoltenberg \\ University of Nebraska-Lincoln, sstoltenberg2@unl.edu
}

Follow this and additional works at: https://digitalcommons.unl.edu/psychfacpub

Part of the Genetics Commons, and the Psychology Commons

Christ, Christa C.; Watkins, Laura E.; DiLillo, David; and Stoltenberg, Scott F., "Alcohol Intoxication Moderates the Association between a Polygenic Risk Score and Unprovoked Intimate Partner Aggression" (2018). Faculty Publications, Department of Psychology. 946.

https://digitalcommons.unl.edu/psychfacpub/946

This Article is brought to you for free and open access by the Psychology, Department of at DigitalCommons@University of Nebraska - Lincoln. It has been accepted for inclusion in Faculty Publications, Department of Psychology by an authorized administrator of DigitalCommons@University of Nebraska - Lincoln. 
Published in Journal of Family Violence 33:1 (January 2018), pp. 83-94; doi: 10.1007/s10896-017-9908-5 Copyright (C) 2017 Springer Science+Business Media. Used by permission.

Published online February 2, 2017.

\title{
Alcohol Intoxication Moderates the Association between a Polygenic Risk Score and Unprovoked Intimate Partner Aggression
}

\author{
Christa C. Christ, ${ }^{1}$ Laura E. Watkins,${ }^{2}$ David DiLillo, ${ }^{3}$ \\ and Scott F. Stoltenberg 3,4
}

1. Department of Psychology, Hamilton College, Clinton, New York, USA

2. U.S. Department of Veterans Affairs National Center for Posttraumatic Stress Disorder, Clinical Neurosciences Division, VA Connecticut Healthcare System and Department of Psychiatry, Yale School of Medicine, Boston, Massachusetts, USA

3. Department of Psychology, University of Nebraska-Lincoln, Lincoln, Nebraska, USA

4. Center for Brain, Biology, and Behavior, University of Nebraska-Lincoln, Lincoln, Nebraska, USA

Corresponding author - Christa C. Christ, email cchrist@hamilton.edu

\begin{abstract}
Despite evidence that genetic variation contributes to aggression, few studies have examined how genetic variation contributes to IPA specifically. In the current study, 69 couples from a Midwestern university completed self-report measures of IPA, childhood trauma exposure, and hazardous alcohol use, and were randomly assigned to consume either a placebo or alcohol beverage before participating in an analogue aggression task against their partner. Genetic risk (i.e., association with lower transcriptional efficiency) for aggression was measured with a polygenic risk score (PRS) created from four polymorphisms (HTR1B rs13212041, HTR2B rs6437000, 5-HTTLPR, and MAOA uVNTR). Among individuals with a low PRS, individuals who consumed alcohol $(\mathrm{BrAC}=0.07 \%)$ showed greater unprovoked IPA than individuals who consumed a placebo. Findings contribute to our limited understanding regarding the etiology of IPA and suggest that individuals who have increased transcriptional activity in certain serotonin system genes may be at higher risk of IPA when intoxicated.
\end{abstract}

Keywords: IPA, MAOA, 5-HTTLPR, HTR1B, HTR2B 
Intimate partner aggression (IPA) includes behaviors intended to cause harm against a relationship partner, such as sexual violence, stalking, physical violence, psychological aggression, and control of sexual health. About $29 \%$ of college students report perpetration of IPA within the past year (Elmquist et al. 2016; Straus 2004). IPA is also a considerable concern among heavy-drinking college students, with more than half reporting perpetrating either physical or psychological aggression against their partner (Fossos et al. 2007). Victims of IPA are more likely to experience adverse outcomes, such as mental health symptoms, high blood pressure, chronic pain, and asthma (Black et al. 2011). Identifying biological and environmental risk factors of IPA may help to better inform prevention efforts.

Genetic, neurobiological, and psychosocial factors are associated with aggression (Rossell and Siever 2015). In the genetic realm, polymorphisms (i.e., genetic variations) that affect the regulation of serotonin neurotransmission are associated with individual differences in aggressive behavior (Craig and Halton 2009). In particular, four genetic variants that impact serotonergic neurotransmission are strong candidates for contributing to individual differences in aggressive behavior. First, the serotonin $1 \mathrm{~b}\left(5-\mathrm{HT}_{1 \mathrm{~B}}\right)$ receptor, encoded by the HTR1B gene, regulates the release of serotonin from the neuron (Hartig 2000). The HTR1B A allele (i.e., one variant of the polymorphism) of the single nucleotide polymorphism (SNP), rs13212041, results in decreased gene transcription (i.e., copying of DNA into mRNA before translation into protein) and is associated with increased self-reported conduct disorder behaviors (Jensen et al. 2009), as well as greater self-reported anger and hostility in men (Conner et al. 2010). Second, the serotonin transporter, encoded by the SLC6A4 gene, is responsible for the reuptake (i.e., absorption) of serotonin back into the presynaptic neuron (Ramamoorthy et al. 1993). The $S$ allele of variable number tandem repeat (VNTR) polymorphism of SLC6A4, 5-HTTLPR, lowers gene transcription, results in fewer serotonin transporters (Hu et al. 2006), and is typically associated with higher aggressiveness (Beitchman et al. 2006; Gerra et al. 2005; Gonda et al. 2009; Retz et al. 2004) compared to the $\mathrm{L}$ allele. Third, the serotonin $2 \mathrm{~b}\left(5-\mathrm{HT}_{2 \mathrm{~B}}\right)$ receptor, encoded by the HTR $2 B$ gene, modulates serotonin reuptake (Launay 2006). The HTR2B A allele of SNP rs6437000 is associated with lower gene expression and increased aggressive behavior (Bevilacqua et al. 2011). Finally, monoamine oxidase A (MAOA) is an enzyme that breaks down serotonin (Weyler et al. 1990). A $M A O A$ gene polymorphism, MAOA uVNTR, affects gene transcription (Sabol et al. 1998), with the low activity variants (i.e., lower rate of gene transcription) typically being associated with increased aggression (Alia-Klein et al. 2008; Gallardo-Pujol et al. 2013; McDermott et al. 2009).

Although the evidence presented above supports an association between lower efficiency in serotonin neurotransmission and increased aggression, there is evidence for 5-HTTLPR and MAOA uVNTR to suggest higher efficiency in serotonin neurotransmission increases aggression (Nobile et al. 2007; Verhoeven et al. 2012). Genetic polymorphisms in other neurotransmitter systems (e.g., dopamine), type of aggression, induction method of aggression, and environmental factors modify the association between serotonin and aggression, possibly accounting for the inconsistencies in previous literature and reinforcing the inclusion of potential modifiers in genetic research (Carrillo et al. 2009; Byrd and Manuck 2014). 
The contribution of genetic variation to IPA has received limited attention. Yet, a twin study found that $16 \%-22 \%$ of the total variance in physical and psychological IPA was accounted for by genetic variation, respectively (Hines and Saudino 2004), suggesting that genetic factors should be considered when investigating the etiology of IPA. Further, there have been two studies that examined the association between specific genetic polymorphisms and IPA. Foshee et al. (2015) found that the L allele of 5-HTTLPR predicted higher self-reported levels of dating violence perpetration during greater self-reported alcohol consumption. Also, the low activity allele of $M A O A$ uVNTR predicted higher dating violence, but the effect was not moderated by self-reported alcohol consumption. A higher polygenic risk score (PRS) composed of the variants MAOA uVNTR and 5-HTTLPR (higher PRS corresponds to lower gene expression) predicted increased psychological aggression, physical assault, and violence resulting in injury (Stuart et al. 2014b). As suggested by Stuart et al. (2014a, b), our current study aims to extend previous research by including additional genetic polymorphisms (i.e., rs6437000, rs13212041) in a PRS and investigating potential moderating effects of alcohol intoxication, and experiences of childhood trauma.

Alcohol intoxication increases aggressive behavior in experimental studies (Chermack and Taylor 1995; Duke et al. 2011; Giancola et al. 2012). Alcohol use has also been shown to increase risk for IPA perpetration (Devries et al. 2013), with 35\% of IPA events co-occurring with alcohol use (Reingle et al. 2014). Both the $S$ allele of 5-HTTLPR and low function variants of MAOA uVNTR have been associated with increased alcohol-related aggression (Hallikainen et al. 1999; Tikkanen et al. 2009). An association between self-reported alcohol consumption and IPA was stronger for individuals who have two copies of the L allele of 5-HTTLPR, although no such association was found with MAOA uVNTR (Foshee et al. 2015). It is currently unknown whether acute alcohol intoxication will moderate the effect of genetic risk on IPA.

Another potential moderating environmental factor on IPA is childhood trauma exposure, because both men and women who experienced childhood trauma are more likely to perpetrate IPA (Eckhardt et al. 2008; McKinney et al. 2009). Additionally, prisoners (i.e., incarcerated individuals) who had a low-activity $M A O A$ uVNTR genotype reported higher aggression if they experienced childhood physical neglect compared to prisoners who did not experience physical neglect during their childhood; this effect was attenuated for prisoners who had a high-activity genotype (Gorodetsky et al. 2014). Further, male rhesus monkeys that carry a 5-HTTLPR $S$ allele were more aggressive to social threat when exposed to early adversity (i.e., separation from mother 1-2 days after birth, nurseryreared) (Schwandt et al. 2010). The current study aims to extend these findings by examining the impact of the interaction between childhood trauma experiences and polygenic risk on IPA.

Our current study seeks to address the limited attention to the genetic contribution to IPA, particularly in the context of various environmental and situational factors with established links to IPA (i.e., childhood trauma, alcohol intoxication). The first goal of the current study was to replicate previous findings by examining the association between the same PRS used by Stuart et al. (PRS1; MAOA uVNTR \& 5-HTTLPR), and self-reported 
physical and psychological aggression. Secondly, to extend previous findings, we will examine whether this association remains when (1) additional polymorphisms are added to the PRS (PRS2; rs6437000 \& rs13212041), and (2) behavioral measures of IPA are used. Lastly, we will examine the moderating effect of alcohol intoxication, experiences of childhood trauma, and gender on the association between PRS and IPA. We hypothesize that there will be a positive linear relationship between PRS and IPA, and that this relationship will be significantly more positive in individuals who experienced childhood trauma and with increased alcohol use (i.e., higher self-report or alcohol experimental condition). Although the association between MAOA uVNTR and aggression is stronger in males (Byrd and Manuck 2014), perpetration of intimate partner aggression among college students appears to be equivalent across men and women (Cercone et al. 2005). Therefore, no a priori hypotheses for the moderating effect of gender were made.

\section{Method}

\section{Participants}

Sixty-nine couples $(N=138)$ were recruited from a large Midwestern University to participate in the current study. The majority of participants (age $M=23.4, S D=2.5$, range $=21-32$ ) were undergraduate students $(48.1 \%)$, while the remaining participants were graduate students $(23.4 \%)$ or not students $(27 \%)$. Participants primarily identified as European-American $(87 \%)$, while the remainder identified as Latino, Hispanic, or Spanish (9.5\%), Asian or Pacific Islander (5.8\%), African American or Black (2.2\%), American Indian, Native American, or Alaskan Native (.7\%), or "other" (3.6\%; participants were allowed to pick more than one category so percentages exceed $100 \%$ ). Participants had been in a relationship for an average of 32 months $(S D=23.3$, range $=4-102)$ and described their relationship as dating $(44.9 \%)$, dating and living together $(24.6 \%)$, married or marriage-like $(19.7 \%)$, or engaged $(10.1 \%)$. The majority of participants described themselves as straight $(94.2 \%), 2.9 \%$ identified as gay (male), 1.5\% identified as lesbian, and 1.5\% identified as bisexual. Details about participant recruitment have been presented elsewhere (Watkins et al. 2015).

\section{Laboratory Tasks and Measures}

\section{Analogue Aggression Task (AAT)}

A competitive computer reaction time task based on the Taylor Aggression Paradigm (Taylor 1967) was used as a behavioral measurement of IPA. The Taylor Aggression Paradigm is a widely used paradigm for observing aggressive behaviors in laboratory studies. It is a reliable and valid (i.e., positively correlated with self-report measures of interpersonal aggression) measure of aggressive behavior for both men and women (Giancola and Chermack 1998; Hoaken and Phil 2000). Participants were informed that they would play a reaction time game against their intimate partner, while in reality they were playing a preprogrammed computer. Participants were asked to complete a series of 25 trials in which they pressed a button as quickly as possible after an onscreen stimulus changed color. Before each trial, participants designated a length (from 0 to $5 \mathrm{~s}$ ) and volume (from 0 to 10) of white noise to ostensibly be blasted over the headphones of their partner on trials that 
they won and their partner lost. Selecting a volume level of 0 produces no sound, providing a non-aggressive option. The 1-10 volume level options range from 60 to $105 \mathrm{~dB}$ in 5-dB increments. Scores for each trial were calculated by averaging the noise intensity and duration. Trial 1 is considered a measure of unprovoked aggression because the participant has not yet received a noise blast (Reidy et al. 2011) and has been used in numerous studies rather than later trials, which may reflect reciprocation (Denson et al. 2010; DeWall et al. 2007). Trial 2 is considered a measure of retaliatory aggression (Wilkowski et al. 2010) because it occurred after a blast of maximum intensity perceived to come from a partner and therefore was an indication of how a person might respond after provocation (being aggressed against by their partner). Trial 2 is also the only trial in which all participants have received the maximum length and volume of noise blast and is therefore considered the purest measure of retaliatory aggression.

\section{Self-Reported Physical and Psychological Aggression}

History of IPA perpetration was assessed with the 12-item Physical Assault subscale (e.g., "kicked my partner"; $\alpha=.69$ ) and the 8-item Psychological Aggression subscale (e.g., "shouted or yelled at my partner"; $\alpha=.63$ ) from the Conflict Tactics Scale - Revised (CTS2; Straus et al. 1996). Participants indicated the frequency at which they perpetrated each aggressive behavior against their partner during the previous six months using an 8-point scale (from $1=$ never to $8=$ more than 20 times). Item responses were summed to create a total IPA score, with higher scores indicating more acts of IPA.

\section{Alcohol Use Disorders Identification Test (AUDIT)}

Hazardous alcohol use was assessed with the 10-item AUDIT (Saunders et al. 1993). Each item is measured on a 5-point scale and addressed frequency and quantity of drinking over the past year. Responses to each item were summed to create a total score $(\alpha=.44$; low alpha coefficient is most likely the result of using a limited range of scores as inclusion criteria).

\section{Childhood Trauma Questionnaire}

Five types of childhood trauma were measured with the 28-item Childhood Trauma Questionnaire (CTQ; Bernstein and Fink 1998). Severity of emotional abuse, physical abuse, sexual abuse, emotional neglect, and physical neglect were measured on a 5-point scale (from $1=$ never to $5=$ very often). Responses to each item were summed to create a total score, which could range from 25 to $125(\alpha=.75)$.

\section{Procedures}

The Institutional Review Board approved all procedures. Participants were asked to refrain from drinking alcohol and using recreational drugs for $24 \mathrm{~h}$, and from eating four hours prior to their scheduled appointment time. Upon arrival, partners were taken to separate rooms where they gave written informed consent. Each participant was randomly assigned to drink either an alcohol-containing or a placebo beverage. The alcohol administration details for the current study are presented elsewhere (Watkins et al. 2015). Participants completed self-report measures and then provided buccal cells for genotyping. As 
part of the larger study, participants were asked to recall an anger-eliciting event for two minutes with instructions to utilize an assigned cognitive emotion regulation strategy (i.e., rumination, reappraisal, or uninstructed) prior to completing the analogue aggression task. The assigned cognitive emotion regulation strategy was used as a covariate in the reported analyses with the analogue aggression task because it was not directly related to the current study's research hypotheses. After completion of data collection, participants watched film clips of waves on a beach in order to increase feelings of contentment and decrease any residual anger (Gross and Levenson 1995). Participants were then fully debriefed and were provided an opportunity to ask questions. Participants who received alcohol were fully debriefed a second time once their $\mathrm{BrAC}$ reached $.03 \%$ or lower and then either had someone pick them up or took a study-provided taxi.

\section{Genotyping}

DNA was extracted from buccal cells (i.e., cheek cells) using the PURGENE DNA Isolation Kit Protocol (Qiagen Corp., Valencia, CA, USA). HTR1B rs13212041 and HTR2B rs6437000 were amplified using Taqman SNP Genotyping Assays following standard manufacturers protocols (Applied Biosystems, Foster City, California). ABI Sequence Detection Software used end point FAM and VIC fluorescence levels to determine genotypes (Applied Biosystems, Foster City, California). The call rate for rs13212041 and rs6437000 were $97.1 \%$ and 100\%, respectively. Both rs13212041 (Minor Allele Frequency [MAF]: $C=.21$ ) and rs6437000 (MAF: $\mathrm{A}=.33$ ) were in Hardy-Weinberg Equilibrium (HWE); $\chi^{2}=.36, p>.05$ and $\chi^{2}=3.20$, $p>.05$, respectively.

5-HTTLPR was amplified using previously established methods (Wendland et al. 2006). Polymerase chain reaction (PCR) product was separated by electrophoresis on a $2.5 \%$ agarose gel and visualized under UV light with SybrSafe stain. Independent calls were made by three trained researchers and compared to determine the final genotype. The call rate for 5-HTTLPR was 94.9\% (MAF: $S=.49)$ and the allele frequency was in HWE $\left(\chi^{2}=.61, p>.05\right)$.

PCR to amplify MAOA uVNTR was performed in $25 \mu \mathrm{l}$ reactions containing $20 \mathrm{ng}$ of DNA, $1 X$ GoTaq Master Mix (Promega, Madison, Wisconsin, USA), $10 \mu \mathrm{M}$ of each primer (5-TGCTCCAGAAACATGAGCAC-3' and 5'-TAGACTTGGGGATCCGACTG-3'), and $.25 \mu \mathrm{L}$ DMSO. Cycling conditions consisted of 5-min initial denaturation at $95^{\circ} \mathrm{C}$ followed by 35 cycles of (1) $30 \mathrm{~s}$ at $95^{\circ} \mathrm{C}$, (2) $30 \mathrm{~s}$ at $55^{\circ} \mathrm{C}$, and (3) $40 \mathrm{~s}$ at $72^{\circ} \mathrm{C}$, and a final extension for $10 \mathrm{~min}$ at $72^{\circ} \mathrm{C}$. PCR product was separated by electrophoresis on a $3 \%$ agarose gel and visualized under UV light with SybrSafe stain. Independent calls were made by two trained researchers and compared to determine the final genotype. Two, 3, or 5 repeat alleles (2R, 3R, \& 5R, respectively) were categorized as "high activity" (HA), while 3.5 or 4 repeat alleles (3.5R \& $4 R$, respectively) were categorized as "low activity" (LA) (Sabol et al. 1998). Due to low inter-rater reliability on samples initially scored as a 3.5R genotype, the samples were rerun using an alternative set of primers (5-ACAGCCTGACCGTGGAGAAG-3' and 5'-AGGCTTACCTCGCAGGCAAG-3') that produced greater distinction between the bands. The PCR components and visualization by gel electrophoresis was the same as the first set of primers. PCR cycling conditions consisted of 10-min initial denaturation at $95^{\circ} \mathrm{C}$ followed by 35 cycles of (1) $1 \mathrm{~min}$ at $95^{\circ} \mathrm{C}$, (2) $1 \mathrm{~min}$ at $55^{\circ} \mathrm{C}$, and (3) $2 \mathrm{~min}$ at $72^{\circ} \mathrm{C}$, and a final extension for $10 \mathrm{~min}$ at $72^{\circ} \mathrm{C}$. The call rate for $M A O A$ uVNTR was $92.8 \%$. Genotypes 
were grouped based on functional expression: high activity (3.5R and $4 \mathrm{R})$ and low activity $(2 \mathrm{R}, 3 \mathrm{R}$, and $5 \mathrm{R})$. The allele frequency for females (LL: .26, LH: .44, HH: .30) was in HWE $\left(\chi^{2}=.94, p>.05\right)$. HWE is not calculated for males (L: .40, H: .60) because MAOA uVNTR is an X-linked gene.

Ten percent of samples were re-genotyped to assess genotyping accuracy, and no discrepancies were found. Allele frequencies for each polymorphism are consistent with European-American ancestry. Genotypes were assigned either 0,1 , or 2 based on its impact on serotonergic transmission and association with aggressive behavior as indicated by previous literature, where higher scores corresponded to higher expected risk for aggression (rs13212041 A/A = 0, A/G =1, G/G = 2; rs6437000 C/C = 0, A/C = 1, A/A = 2; 5-HTTLPR L/L $=0, \mathrm{~L} / \mathrm{S}=1, \mathrm{~S} / \mathrm{S}=2 ; M A O A \mathrm{uVNTR} \mathrm{HA} / \mathrm{HA}=0, \mathrm{HA} / \mathrm{LA}=1, \mathrm{LA} / \mathrm{LA}=2)$. In order to include those who did not have a genotype score for all four variants $(N=18)$, the PRS score was calculated by averaging the genotype scores, resulting in a score for each participant that ranged from 0 to 1 .

\section{Analytic Approach}

One participant become ill during the study and was therefore excluded from our analyses; thus data from 137 individuals (68 women, 69 men) was used in analyses. Self-reported psychological and physical aggression using the CTS2 as well as a combined score of white noise level and duration for Trial 1 and Trial 2 from the AAT were used as measures of IPA. For self-report physical and psychological aggression, PRS, hazardous alcohol use (AUDIT), gender $(0=$ female, $1=$ male), childhood trauma, and all two-way interactions were tested in a regression model, controlling for age, and ethnicity ( $0=$ European-American, $1=$ Non-European-American). For intimate partner aggression as measured by Trial 1 and Trial 2 of the AAT, PRS, study condition ( $0=$ alcohol, $1=$ placebo), gender, childhood trauma, and all two-way interactions were tested in a regression model controlling for age, ethnicity, and instructions.

Due to the limited number of participants endorsing any physical IPA perpetration $(N=$ $121 ; 88.3 \%)$, this outcome was coded as occurred (1) or not occurred (0), and a logistic model was used. A general linear model was used for psychological aggression. To account for the skewness, a log-normal regression model was performed for Trial 1 using PROC GLIMMIX in SAS. Because Trial 2 was specifically skewed by a large number of individuals selecting the highest possible volume-duration score, a censored regression model using PROC QLIM in SAS was used to account for the fact that some individuals would have potentially selected a higher volume-duration if that had been an option. Results are presented with uncorrected $p$-values.

Because of the dyadic nature of the data, for each model we tested whether a correlation between the residuals of partners' outcomes improved model fit. For both physical and psychological IPA, this correlation did result in better model fit, $-2 \Delta \operatorname{LL}(d f=1)=8.8, p<.01$ and $-2 \Delta \mathrm{LL}(d f=1)=62.6, p<.001$, respectively, and thus we kept the correlation in the model. For both white noise trials, models with the additional correlation did not fit better than models without the correlation, $-2 \Delta \mathrm{LL}(d f=1)=1.6, p=.21$ and $-2 \Delta \mathrm{LL}(d f=1)=2.7$, $p=.10$, respectively and thus these correlations were not kept in these models. 


\section{Results}

\section{Couple-Reported IPA}

Results for couple-reported physical IPA are displayed in Table 1. For PRS1 and couplereported physical IPA, the scale of the PRS1 score was multiplied by 100 to make the variance estimate less prone to numerical instability given the small estimates in analyses. When using either PRS1 or PRS2, there were no significant predictors of couple-reported physical IPA. Results for couple-reported psychological IPA are displayed in Table 1. When using PRS1, a main effect was found for age $(b=-.09, p=.049)$, such that as age increased, psychological IPA decreased (see Table 1). In addition, the interaction between childhood trauma and hazardous alcohol use significantly predicted psychological IPA ( $b$ $=-.01, p=.01$ ), such that as hazardous alcohol use increased, the relationship between childhood trauma and IPA became less positive (see Fig. 1). For instance, among individuals one standard deviation below the mean on the AUDIT, the relationship between childhood trauma and psychological aggression was significantly positive $(b=.07, p=.03)$, whereas for individuals one standard deviation above the mean on the AUDIT, the relationship between childhood trauma and psychological IPA was not significant $(b=.01, p=$ $.61)$. When using the PRS2, the main effect for age was significant $(b=-.10, p=.04)$, however, the interaction between childhood trauma and hazardous alcohol use was not significant (see Table 1).

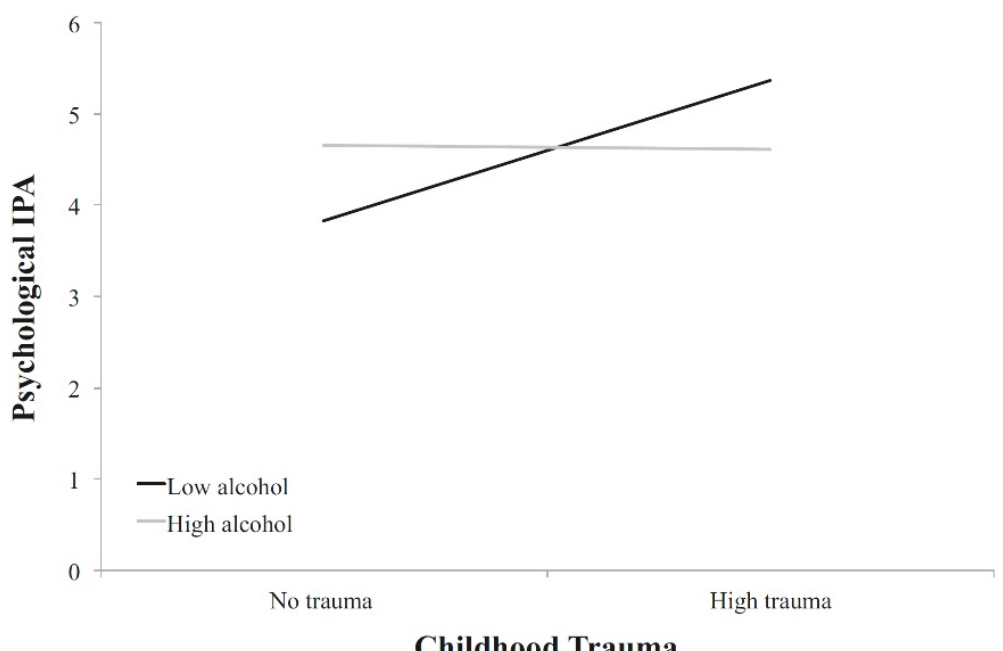

Figure 1. Moderating effect of childhood trauma on the association between alcohol use and (including MAOA uVNTR \& 5-HTTLPR) self-reported psychological aggression. Among individuals with AUDIT scores one standard deviation below the mean, the relationship between childhood trauma and psychological aggression was significantly positive. And among those with AUDIT scores one standard deviation above the mean, the relationship between childhood trauma and psychological IPA was not significant. 


\begin{tabular}{|c|c|c|}
\hline Predictor & Physical IPA Estimate (SE) & Psychological IPA Estimate $(S E)$ \\
\hline \multicolumn{3}{|l|}{ PRS Including MAOA \& 5-HTTLPR } \\
\hline Intercept & $3.499(6.593)$ & $3.581(1.158)$ \\
\hline Race/Ethnicity $($ Non-White $=1)$ & $1.267(1.282)$ & $-0.071(0.271)$ \\
\hline Age & $-0.252(0.271)$ & $-0.095(0.048)^{*}$ \\
\hline Polygenic Risk Score (PRS) & $-0.007(0.019)$ & $0.481(0.342)$ \\
\hline Gender $($ Male $=1)$ & $0.308(2.567)$ & $0.678(0.440)$ \\
\hline Childhood Trauma & $0.111(0.221)$ & $0.090(0.039)^{*}$ \\
\hline Hazardous Alcohol Use & $-0.880(0.690)$ & $0.153(0.107)$ \\
\hline PRS * Gender & $-0.018(0.019)$ & $-0.534(0.302)$ \\
\hline PRS * Childhood Trauma & $-0.001(0.001)$ & $-0.015(0.015)$ \\
\hline PRS * Hazardous Alcohol Use & $0.004(0.005)$ & $-0.023(0.077)$ \\
\hline Gender * Childhood Trauma & $-0.162(0.137)$ & $-0.013(0.021)$ \\
\hline Gender * Hazardous Alcohol Use & $0.440(0.528)$ & $-0.088(0.074)$ \\
\hline $\begin{array}{l}\text { Childhood Trauma * Hazardous Alcohol } \\
\text { Use }\end{array}$ & $-0.012(0.037)$ & $-0.015(0.006)^{*}$ \\
\hline \multicolumn{3}{|l|}{ PRS Including All Four Polymorphisms } \\
\hline Intercept & $8.784(17.951)$ & $3.600(1.183)$ \\
\hline Race/Ethnicity (Non-White $=1$ ) & $3.268(3.046)$ & $-0.054(0.272)$ \\
\hline Age & $-0.720(0.708)$ & $-0.100(0.047)^{*}$ \\
\hline Polygenic Risk Score (PRS) & $3.824(7.143)$ & $0.741(0.412)$ \\
\hline Gender $($ Male $=1)$ & $-7.285(9.581)$ & $0.738(0.476)$ \\
\hline Childhood Trauma & $-0.407(0.745)$ & $0.072(0.043)$ \\
\hline Hazardous Alcohol Use & $-0.463(2.136)$ & $0.163(0.143)$ \\
\hline PRS * Gender & $-1.752(5.075)$ & $-0.699(0.412)$ \\
\hline PRS * Childhood Trauma & $0.676(0.638)$ & $-0.015(0.027)$ \\
\hline PRS * Hazardous Alcohol Use & $-1.568(1.982)$ & $-0.075(0.110)$ \\
\hline Gender * Childhood Trauma & $-0.338(0.316)$ & $-0.018(0.021)$ \\
\hline Gender * Hazardous Alcohol Use & $2.483(1.870)$ & $-0.044(0.071)$ \\
\hline $\begin{array}{l}\text { Childhood Trauma * Hazardous Alcohol } \\
\text { Use }\end{array}$ & $0.014(0.082)$ & $-0.011(0.006)$ \\
\hline
\end{tabular}

\section{Unprovoked IPA}

For Trial 1 (unprovoked aggression) and PRS1, there was a significant effect of condition $(b=.59, p=.047)$, such that those in the alcohol condition had higher Trial 1 aggression scores than those in the placebo condition (see Table 2). When using the PRS2, several additional significant predictors emerged (see Table 2). A significant interaction was found between alcohol condition and PRS2 $(b=-.68, p=.02)$, such that aggression scores increased less positively as PRS2 increased for those in the alcohol condition compared to those in the placebo condition (see Fig. 2). The significant interaction indicates the association between polygenic risk and aggression was significantly different for those in the alcohol condition compared to individuals in the placebo condition, but the association between polygenic risk and aggression itself was not significant for individuals in either condition. 
More specifically, PRS2 had a nonsignificant positive impact on aggression among individuals in the placebo condition $(b=.38, p=.23)$ and a nonsignificant negative impact on aggression among individuals in the alcohol condition $(b=-.30, p=.26)$. When examining the simple effects of condition on aggression at both low $($ PRS2 $=0)$ and high (PRS2 =1) polygenic risk, those in the alcohol condition had significantly higher trial 1 aggression scores compared to those in the placebo condition $(b=.95, p=.02)$ for individuals with a low PRS2. Among individuals with high PRS2, the impact of alcohol on aggression was not significant $(b=-.40, p=.26)$.

\begin{tabular}{|c|c|c|}
\hline Predictor & Trial 1 Estimate $(S E)$ & Trial 2 Estimate $(S E)$ \\
\hline \multicolumn{3}{|l|}{ PRS Including MAOA \& 5-HTTLPR } \\
\hline Intercept & $0.724(0.642)$ & $6.854(3.764)$ \\
\hline No Instruction vs. Reappraisal (Reappraisal = 1) & $0.129(0.146)$ & $1.141(0.880)$ \\
\hline No Instruction vs. Rumination (Rumination $=1$ ) & $0.177(0.150)$ & $1.213(0.890)$ \\
\hline Race/Ethnicity (Non-White $=1$ ) & $0.010(0.189)$ & $0.628(1.248)$ \\
\hline Age & $-0.013(0.026)$ & $-0.159(0.160)$ \\
\hline Polygenic Risk Score (PRS) & $0.180(0.217)$ & $0.201(1.118)$ \\
\hline Gender $($ Male $=1)$ & $0.371(0.322)$ & $0.851(1.675)$ \\
\hline Childhood Trauma & $0.020(0.018)$ & $0.090(0.115)$ \\
\hline Placebo vs. Alcohol Condition $($ Alcohol $=1)$ & $0.590(0.293)^{*}$ & $2.096(1.636)$ \\
\hline PRS * Gender & $-0.012(0.223)$ & $-0.149(1.255)$ \\
\hline PRS * Childhood Trauma & $-0.001(0.009)$ & $0.001(0.061)$ \\
\hline PRS * Placebo vs. Alcohol Condition & $-0.365(0.200)$ & $-1.367(1.284)$ \\
\hline Gender * Childhood Trauma & $-0.011(0.014)$ & $-0.223(0.099)^{*}$ \\
\hline Gender * Placebo vs. Alcohol Condition & $-0.074(0.254)$ & $-2.777(0.059)$ \\
\hline Childhood Trauma * Placebo vs. Alcohol Condition & $-0.013(0.016)$ & $0.032(0.763)$ \\
\hline \multicolumn{3}{|l|}{ PRS Including All Four Polymorphisms } \\
\hline Intercept & $0.302(0.700)$ & $3.783(4.034)$ \\
\hline No Instruction vs. Reappraisal (Reappraisal $=1$ ) & $0.125(0.141)$ & $1.152(0.864)$ \\
\hline No Instruction vs. Rumination (Rumination $=1$ ) & $0.183(0.146)$ & $1.166(0.876)$ \\
\hline Race/Ethnicity (Non-White $=1$ ) & $-0.026(0.896)$ & $0.477(1.281)$ \\
\hline Age & $-0.006(0.024)$ & $-0.143(0.155)$ \\
\hline Polygenic Risk Score (PRS) & $0.379(0.315)$ & $2.555(1.633)$ \\
\hline Gender $($ Male $=1)$ & $0.586(0.407)$ & $3.321(2.257)$ \\
\hline Childhood Trauma & $0.016(0.021)$ & $0.179(0.152)$ \\
\hline Placebo vs. Alcohol Condition $($ Alcohol $=1)$ & $0.954(0.394)^{*}$ & $3.778(2.197)$ \\
\hline PRS * Gender & $-0.167(0.297)$ & $-2.346(1.841)$ \\
\hline PRS * Childhood Trauma & $0.007(0.016)$ & $-0.070(0.115)$ \\
\hline PRS * Placebo vs. Alcohol Condition & $-0.676(0.296)^{*}$ & $-2.694(1.896)$ \\
\hline Gender * Childhood Trauma & $-0.016(0.014)$ & $-0.229(0.099)^{*}$ \\
\hline Gender * Placebo vs. Alcohol Condition & $-0.067(0.258)$ & $2.784(1.480)$ \\
\hline Childhood Trauma * Placebo vs. Alcohol Condition & $-0.014(0.015)$ & $0.010(0.102)$ \\
\hline
\end{tabular}




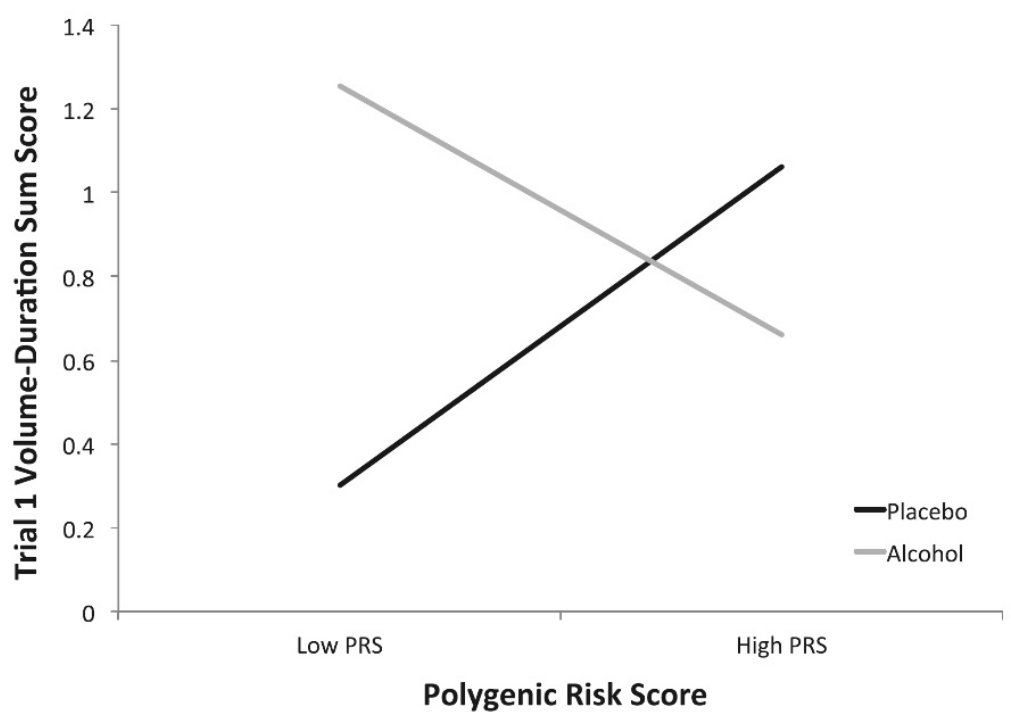

Figure 2. Moderating effect of alcohol consumption on the association between PRS and (including all four polymorphisms) unprovoked aggression (Trial 1). At low genetic risk, unprovoked aggression is higher for individuals who consumed alcohol than for those who consumed a placebo. Y-axis corresponds to log trial 1 volume-duration sum scores.

\section{Retaliatory IPA}

For Trial 2 (retaliatory aggression) and PRS1 (see Table 2), the interaction between gender and childhood trauma was found to be a significant predictor of aggression against one's partner $(b=-.22 ; p=.02)$. Examination of the simple effects indicated, among women, child trauma was not a significant predictor of aggression $(b=.09, p=.43)$, however, among men, child abuse negatively predicted aggression $(b=-.16, p=.03)$. When using PRS2, the interaction between gender and childhood trauma was still found to significantly predict aggression against one's partner $(b=-.23 ; p=.02)$. In contrast to the analysis examining PRS1, when using PRS2 (see Table 2), child abuse was not related to IPA among women $(b=.18$, $p=.18)$ or men $(b=-.05, p=.13)$. Figure 3 displays the interactions for trial 2 IPA. 

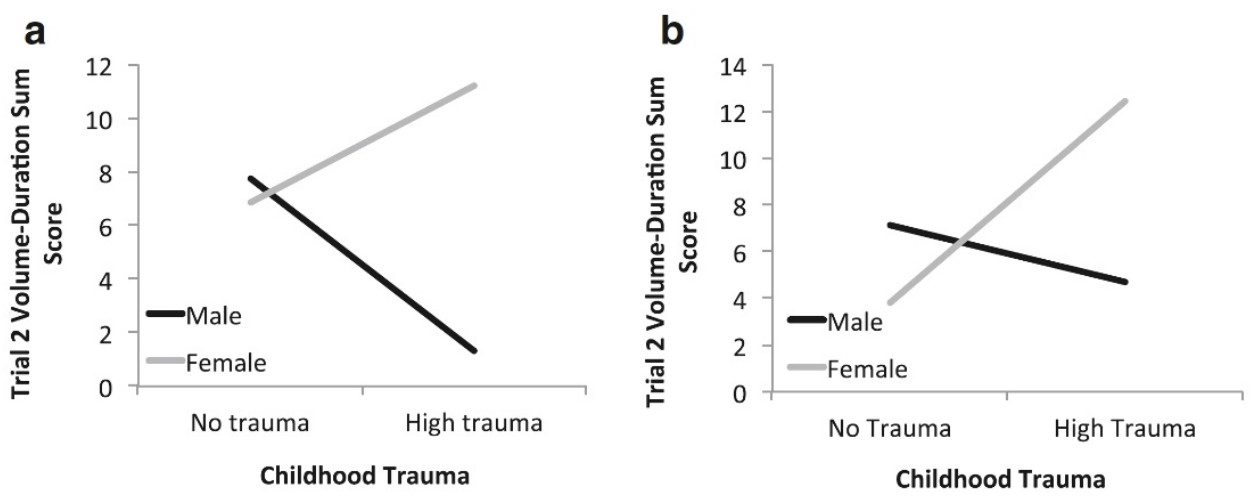

Figure 3. On Trial 2 (a) for analyses including the PRS1, there was no association between childhood trauma and retaliatory aggression for women, and there was a negative association between childhood trauma and retaliatory aggression for men, controlling for all other variables in the model; (b) for analyses including the PRS2, there was no significant association between childhood trauma and retaliatory aggression for either men or women, controlling for all other variables in the model. Y-axis corresponds to trial 2 volumeduration sum scores adjusted with a censored model.

\section{Discussion}

As hypothesized, we found a significant moderating effect of the PRS (score using all four polymorphisms) on the association between alcohol intoxication and Trial 1 or unprovoked IPA; however, the effect was in the opposite direction than we expected. Specifically, aggression was higher for individuals in the alcohol condition compared to individuals in the placebo condition when PRS was low. The lack of association between alcohol condition and unprovoked aggression for individuals with high genetic risk scores may be partly due to participants being instructed to recall an anger-eliciting event, which increased anger in all participants, prior to their completion of the analogue aggression paradigm. As proposed by the multiple threshold model of IPV (Fals-Stewart and Stappenbeck 2003), alcohol is not necessary to surpass the threshold to engage in violence for individuals who are already above the threshold. Therefore it is possible that individuals at higher genetic risk for aggression who are experiencing current angry affect, may already be above the threshold for violence and subsequently unaffected by alcohol, whereas those with lower genetic risk would be expected to have a higher threshold and be more susceptible to the effects of alcohol. Another possible explanation is that the strong effect of alcohol intoxication on IPA weakens the contribution of genetic variation. This possibility is supported by the findings of Turkheimer et al. (2003) who found that the contribution of genes to intelligence is weakened by socioeconomic status. Alternatively, our genetic risk score was calculated based on previous literature that suggested alleles resulting in lower transcriptional activity are associated with greater general aggression (Alia-Klein et al. 2008; Beitchman et al. 2006; Bevilacqua et al. 2011; Conner et al. 2010; Gallardo-Pujol et al. 2013; Gerra et al. 2005; Gonda et al. 2009; McDermott et al. 2009). However, the one previous study examining the interaction between genetic variation and alcohol on IPA (Foshee et al. 2015) 
suggests the L-allele of SLC6A4 5-HTTLPR (higher transcriptional activity) was associated with higher dating violence when there was higher alcohol consumption. The LL genotype is also associated with early onset alcoholism (Laucht et al. 2009). Although limited, previous research examining associations between genetic variation and IPA as moderated by alcohol use suggests that risk alleles associated with general interpersonal aggression may not be the same as the risk alleles for IPA, particularly in the context of alcohol intoxication.

The polygenic risk scores comprised of MAOA uVNTR \& SLC6A4 5-HTTLPR genotypes or all four polymorphisms were not associated with self-reported physical or psychological aggression. In the current study, we used a college sample and excluded participants with severe levels of aggression or alcohol use. This approach contrasts with Stuart et al. (2014b), who examined men from a batterer intervention program, potentially contributing to the inconsistencies between our findings. The average self-report score of intimate partner aggression (Psychological IPA $M=2.30, S D=1.47$; Physical IPA $M=0.15, S D=0.47$ ) and hazardous alcohol use $(M=5.49, S D=1.92)$ in our study is lower than that reported by Stuart et al. (2014b), which may partly be due to our exclusion of individuals reporting severe IPA or alcohol use. Thus, genetic risk may have a larger effect in individuals who are more aggressive or who have more problematic alcohol use. It is also possible that either our findings or the findings presented by Stuart et al. (2014b) are the result of a Type III error (i.e., correctly rejecting the null hypothesis, but the direction of the effect is false). As a result of low power and small effect sizes, the lack of replication in genetic association studies may be unreasonably high (Duncan and Keller 2011), suggesting that replication of our findings with a larger sample, particularly including individuals with higher rates of aggression and alcohol use, is warranted.

There were also no significant genetic effects on retaliatory aggression. Although genes account for a similar amount of variation (about $40 \%$ ) in both reactive and proactive aggression, with proactive aggression having slightly higher heritability (Brendgen et al. 2006), the genetic stability (Tuvblad et al. 2009) and genetic correlation (Brendgen et al. 2006) between reactive and proactive aggression differ, suggesting that genetic factors that contribute to different forms of aggression may vary. A recent review suggests that aminergic systems regulate both reactive and proactive aggression, however specific mechanism underlying genetic differences across reactive and proactive aggression is still needed (Waltes et al. 2015). Our findings suggest that the polymorphisms included in PRS2 interact with alcohol use to predict unprovoked aggression but not provoked aggression. However, our nonsignificant effect of PRS on provoked aggression may also be due to our small sample size.

In addition to our genetic findings, results also revealed that childhood trauma interacted with other variables to predict IPA. Although women's childhood trauma was unrelated to provoked IPA when examining the PRS with MAOA and 5-HTTLPR, men's childhood trauma was negatively related to provoked IPA, which is inconsistent with previous research (Okuda et al. 2015; Roberts et al. 2011). However, the inclusion of additional polymorphisms (i.e., rs13212041, rs6437000) resulted in a nonsignificant effect of childhood trauma on IPA in males. Ideally, we would have examined the interaction between genetic risk, childhood trauma, and gender on IPA, but were unable to do so because of our small 
sample size. Replication of these results in a larger sample to account for higher order effects is needed before any strong conclusions can be made. In addition, among individuals with low alcohol use, childhood trauma positively predicted psychological IPA, while among individual with higher alcohol use, childhood trauma was not related to psychological IPA. The reason for this finding is unclear, as these two established risk factor are commonly expected to exacerbate each other (i.e., result in a positive interaction). The restricted range of alcohol use in the current study caused by our exclusion criteria (e.g., AUDIT scores of 10 or higher) and the low levels of childhood trauma may have contributed to this unexpected finding.

A strength of our analyses includes the use of polygenic risk, because each polymorphism is likely to account for a small amount of variance (Plomin et al. 2009). By considering multiple polymorphisms, we can examine the collective impact of genetic variation while increasing our statistical power. Our results are also strengthened through the use of an experimental design in which participants are randomly assigned to either an alcohol or placebo condition. This approach allows for the direct observation of alcohol effects on IPA as opposed to using retrospective self-report. Additionally, the use of multiple measures of IPA (i.e., observational, self-report) allows for a greater insight into the complex etiology of IPA. Although we did not find an effect of genetic risk on self-reported IPA or retaliatory aggression, polygenic risk did interact with alcohol to impact unprovoked aggression, suggesting that the contribution of the genes used in our study is specific to unprovoked IPA as opposed to other forms of IPA measured (i.e., retaliatory, self-reported physical and psychological).

Our study also had several limitations. Although four functional polymorphisms (i.e., it produces an observable alteration in the manner in which the gene encodes the protein; Dick et al. 2015) were included in our PRS, which is a greater number than the only previous study to examine the association between a PRS related to serotonin neurotransmission and IPA (Stuart et al. 2014b), these four polymorphisms hardly account for the number of polymorphisms that actually contribute to the variance in IPA. Therefore, additional functional polymorphisms, as they are determined to contribute to increased risk of IPA perpetration, should be included in future studies. Also, the formula used to create our PRS assumes an additive model of the predetermined risk allele in which each polymorphism contributes equally to the risk score, which is likely an oversimplification. As more data become available on the association between genetic polymorphisms and IPA, genetic risk scores should account for the relative contribution of each polymorphism. Although the amount of self-reported childhood trauma in our sample $(M=31.41, S D=8.41)$ was consistent with other college-age samples (Paivio and Cramer 2004; Stein et al. 2008; Wright et al. 2001), it is lower than several studies associating childhood trauma with IPA (Okuda et al. 2015; Roberts et al. 2011). Further examination of the moderating effect of childhood trauma, and particularly different types (e.g., physical vs. psychological) of childhood trauma, on the potential association between polygenic risk and IPA in samples reporting more severe childhood maltreatment may demonstrate a different pattern of results. Lastly, the racial diversity of our sample (i.e., primarily European-American) is consistent with previous research to examine IPA perpetration among college students (Cercone 
et al. 2005; Elmquist et al. 2016). However, future studies would benefit from using a more diverse population, as our findings cannot be generalized to other racial or ethnic groups.

IPA is a growing concern on college campuses. In our sample, $83.2 \%$ reported perpetrating psychological aggression toward their partner while $11.7 \%$ reported perpetrating psychological aggression in the past 6 months. Currently only a few published studies have examined the association between genetic risk and IPA, and our study is the first to examine the association among college students. Thus, our study contributes to the limited knowledge on the possible genetic risk for IPA. Given the large body of research associating genetic risk and general aggression, it is important to consider genetic risk for IPA in order to increase our understanding of IPA and to provide more effective prevention efforts and treatment strategies for IPA perpetrators. Although there is a current disconnect between the basic research of candidate-gene associations and applied research, several studies have demonstrated that genetic risk moderates the effectiveness of prevention programs on various behaviors (e.g., alcohol use, externalizing, problem behaviors; Albert et al. 2015; Brody et al. 2009). A better understanding of genetic effects on intimate partner aggression may help inform more personalized prevention and intervention strategies.

\section{References}

Albert, D., Belsky, D. W., Crowley, D. M., Conduct Problems Prevention Research Group, Bates, J. E., Pettit, G. S., et al. (2015). Developmental mediation of genetic variation in response to the fast track prevention program. Development and Psychopathology, 27, 81-95.

Alia-Klein, N., Goldstein, R. Z., Kriplani, A., Logan, J., Tomasi, D., Williams, B., et al. (2008). Brain monoamine oxidase-a activity predicts trait aggression. Journal of Neuroscience, 28, 5099-5104.

Beitchman, J. H., Baldassarra, L., Mik, H., De Luca, V., King, N., Bender, D., et al. (2006). Serotonin transporter polymorphism and persistent, pervasive childhood aggression. The American Journal of Psychiatry, 163, 1103-1105.

Bernstein, D. P., \& Fink, L. (1998). Childhood trauma questionnaire: A retrospective self-report. San Antonio: The Psychological Corporation.

Bevilacqua, L., Hadjulis, M., Enoch, M.-A., New, A. S., Hodgkinson, C. A., Yuan, Q., et al. (2011). An HTR2B low-expression haplotype predicts risk of cocaine dependence and aggression [meeting abstract]. Biological Psychiatry, 69, 60S-61S.

Black, M. C., Basile, K. C., Breiding, M. J., Smith, S. G., Walters, M. L., Merrick, M. T., et al. (2011). The national intimate partner and sexual violence survey (NISVS): 2010 summary report. Atlanta: National Center for Injury Prevention and Control, Centers for Disease Control and Prevention.

Brendgen, M., Vitaro, F., Boivin, M., Dionne, G., \& Perusse, D. (2006). Examining genetic and environmental effects on reactive versus proactive aggression. Developmental Psychology, 42, 1299-1312.

Brody, G. H., Beach, S. R. H., Philibert, R. A., Chen, Y.-F., \& Murry, V. M. (2009). Prevention effects moderate the association of 5-HTTLPR and youth risk behavior initiation: Gene-environment hypotheses tested via a randomized prevention design. Child Development, 80, 645-661.

Byrd, A. L., \& Manuck, S. B. (2014). MAOA, childhood maltreatment, and antisocial behavior: metaanalysis of a gene-environment interaction. Biological Psychiatry, 75, 9-17.

Carrillo, M., Ricci, L. A., Coppersmith, G. A., \& Melloni Jr., R. H. (2009). The effect of increased serotonergic neurotransmission on aggression: a critical meta-analytical review of preclinical studies. Psychopharmacology, 205, 349-368. 
Cercone, J. J., Beach, S. R. H., \& Arias, H. (2005). Gender symmetry in dating intimate partner violence: does similar behavior imply similar constructs? Violence and Victims, 20, 207-218.

Chermack, S. T., \& Taylor, S. P. (1995). Alcohol and human physical aggression: Pharmacological versus expectancy effects. Journal of Studies on Alcohol, 56, 449-456.

Conner, T. S., Jensen, K. P., Tennen, H., Furneaux, H. M., Kranzler, H. R., \& Covault, J. (2010). Functional polymorphisms in the serotonin 1B receptor gene (HTR1B) predict self-reported anger and hostility among young men. American Journal of Medical Genetics. Part B, Neuropsychiatric Genetics, 153B, 67-78.

Craig, I. W., \& Halton, K. E. (2009). Genetics of human aggressive behaviour. Human Genetics, 126, 101-113.

Denson, T. F., von Hippel, W., Kemp, R., \& Two, L. S. (2010). Glucose consumption decreases impulsive aggression in response to provocation in aggressive individuals. Journal of Experimental Social Psychology, 46, 1023-1028.

Devries, K. M., Child, J. C., Bacchus, L. J., Mak, J., Falder, G., Graham, K., et al. (2013). Intimate partner violence victimization and alcohol consumption in women: a systematic review and metaanalysis. Addiction, 109, 379-391.

DeWall, C. N., Baumeister, R. F., Stilman, T. F., \& Gailliot, M. T. (2007). Violence restrained: effects of self-regulation and its depletion on aggression. Journal of Experimental and Social Psychology, 43, 62-72.

Dick, D. M., Agrawal, A., Keller, M. C., Adkins, A., Aliev, F., Monroe, S., \& Sher, K. J. (2015). Candidate gene-environment interaction research: reflections and recommendations. Perspectives on Psychological Science, 10, 37-59.

Duke, A. A., Giancola, P. R., Morris, D. H., Holt, J. C., \& Gunn, R. L. (2011). Alcohol dose and aggression: another reason why drinking more is a bad idea. Journal of Studies on Alcohol and Drugs, 72, 34-43.

Duncan, L., \& Keller, M. C. (2011). A critical review of the first ten years of measured gene-byenvironment interaction research in psychiatry. American Journal of Psychiatry, 168, 1041-1049.

Eckhardt, C. I., Samper, R. E., \& Murphy, C. M. (2008). Anger disturbances among perpetrators of intimate partner violence: clinical characteristics and outcomes of court-mandated treatment. Journal of Interpersonal Violence, 23, 1600-1617.

Elmquist, J., Wolford-Clevenger, C., Zapor, H., Febres, J., Shorey, R. C., Hamel, J., \& Stuart, G. L. (2016). A gender comparison of motivations for physical dating violence among college students. Journal of Interpersonal Violence, 31, 186-203.

Fals-Stewart, W., Stappenbeck, C. A. (2003). Intimate partner violence and alcohol use: the role of drinking in partner violence and implications for intervention. Family Law Psychology Briefs, 4. from http://www.jmcraig.com/subscribers/archives.htm

Foshee, V. A., Benefield, T. S., Puvanesarajah, S., Reyes, H. L. M., Haberstick, B. C., Smolen, A., et al. (2015). Self-regulatory failure and the perpetration of adolescent dating violence: examining an alcohol use by gene explanation. Aggressive Behavior, 41, 189-203.

Fossos, N., Neighbors, C., Kaysen, D., \& Hove, C. M. (2007). Intimate partner violence perpetration and problem drinking among college students: the roles of expectancies and subjective evaluations of alcohol aggression. Journal of Study on Alcohol and Drugs, 68, 706-713.

Gallardo-Pujol, D., Andres-Pueyo, A., \& Maydeu-Olivares, A. (2013). MAOA genotype, social exclusion and aggression: an experimental test of a gene-environment interaction. Genes, Brain, and Behavior, 12, 140-145. 
Gerra, G., Garofano, L., Castaldini, L., Rovetto, F., Zaimovic, A., Moi, G., et al. (2005). Serotonin transporter polymorphism genotype is associated with temperament, personality traits and illegal drugs use among adolescents. Journal of Neural Transmission, 112, 1397-1410.

Giancola, P. R., \& Chermack, S. T. (1998). Construct validity of laboratory aggression paradigms: a response to Tedeschi and Quigley (1996). Aggression and Violent Behavior, 3, 237-253.

Giancola, P. R., Parrott, D. J., Silvia, P. J., DeWall, C. N., Begue, L., Subra, B., et al. (2012). The disguise of sobriety: unveiled by alcohol in persons with an aggressive personality. Journal of Personality, $80,163-185$.

Gonda, X., Fountoulakis, K. N., Juhasz, G., Rihmer, Z., Lazary, J., Laszik, A., et al. (2009). Association of the $S$ allele of the 5-HTTLPR with neuroticism-related traits and temperaments in a psychiatrically healthy population. European Archives of Psychiatry and Clinical Neuroscience, 259, 106-113.

Gorodetsky, E., Bevilacqua, L., Carli, V., Sarchiapone, M., Roy, A., Goldman, D., \& Enoch, M. A. (2014). The interactive effect of MAOA-LPR genotype and childhood physical neglect on aggressive behaviors in Italian male prisoners. Genes, Brain and Behavior, 13, 543-549.

Gross, J. J., \& Levenson, R. W. (1995). Emotion elicitation using films. Cognition and Emotion, 9, 87-108.

Hallikainen, T., Saito, T., Lachman, H. M., Volavka, J., Pohjalainen, T., Ryynanen, O.-P., et al. (1999). Association between low activity serotonin transporter promoter genotype and early onset alcoholism with habitual impulsive violent behavior. Molecular Psychiatry, 4, 385-388.

Hartig, P. R. (2000). Molecular biology and transductional characteristics of 5-HT receptors. In H. G. Baumgarten \& M. Gothert (Eds.), Serotoninergic neurons and 5-HT receptors in the CNS (pp. 175212). Berlin: Springer.

Hines, D. A., \& Saudino, K. J. (2004). Genetic and environmental influences on intimate partner aggression: a preliminary study. Violence and Victims, 19, 701-718.

Hoaken, P. N. S., \& Phil, R. O. (2000). The effects of alcohol intoxication on aggressive responses in men and women. Alcohol and Alcoholism, 35, 471-477.

Hu, X.-Z., Lipsky, R. H., Zhu, G., Akhtar, L. A., Taubman, J., Greenberg, B. D., et al. (2006). Serotonin transporter promoter gain-of-function genotypes are linked to obsessive-compulsive disorder. The American Journal of Human Genetics, 78, 815-826.

Jensen, K. P., Covault, J., Conner, T. S., Tennen, H., Kranzler, H. R., \& Furneaux, H. M. (2009). A common polymorphism in serotonin receptor $1 \mathrm{~B}$ mRNA moderates regulation by miR-96 and associates with aggressive human behaviors. Molecular Psychiatry, 14, 381-389.

Laucht, M., Treutlein, J., Schmid, B., Blomeye, D., Becker, K., Buchmann, A. F., et al. (2009). Impact of psychosocial adversity on alcohol intake in young adults: moderation by the LL genotype of the serotonin transporter polymorphism. Biological Psychiatry, 66, 102-109.

Launay, J. M. (2006). Serotonin transport and serotonin transporter-mediated antidepressant recognition are controlled by 5-HT2B receptor signaling in serotonergic neuronal cells. The FASEB Journal, 20, 1843-1854.

McDermott, R., Tingley, D., Cowden, J., Frazzetto, G., \& Johnson, D. D. P. (2009). Monoamine oxidase a gene (MAOA) predicts behavioral aggression following provocation. PNAS, 106, 2118-2123.

McKinney, C. M., Caetano, R., Ramisetty-Mikler, S., \& Nelson, S. (2009). Childhood family violence and perpetration and victimization of intimate partner violence: findings from a nation population-based study of couples. Annals of Epidemiology, 19, 25-32.

Nobile, M., Giorda, R., Marino, C., Carlet, O., Pastore, V., Vanzin, L., et al. (2007). Socioeconomic status mediates the genetic contribution of the dopamine receptor D4 and serotonin transporter linked promoter region repeat polymorphisms to externalization in preadolescence. Developmental Psychopathology, 19, 1147-1160. 
Okuda, M., Picazo, J., Olfson, M., Hasin, D. S., Liu, S. M., Bernardi, S., \& Blanco, C. (2015). Prevalence and correlates of anger in the community: results from a national survey. CNS Spectrums, 20, 130-139.

Paivio, S. C., \& Cramer, K. M. (2004). Factor structure and reliability of the childhood trauma questionnaire in a Canadian undergraduate student sample. Child Abuse E Neglect, 28, 889-904.

Plomin, R., Haworth, C. M. A., \& Davis, O. S. P. (2009). Common disorders are quantitative traits. Nature Reviews Genetics, 10, 872-878.

Ramamoorthy, S., Bauman, A. L., Moore, K. R., Han, H., Yang-Feng, T., Chang, A. S., et al. (1993). Antidepressant- and cocaine-sensitive human serotonin transporter: molecular cloning, expression, and chromosomal localization. Proceedings of the National Academy of Sciences of the United States of America, 90, 2542-2546.

Reidy, D. E., Shelley-Tremblay, J. F., \& Lilienfeld, S. O. (2011). Psychopathy, reactive aggression, and precarious proclamations: a review of behavioral, cognitive, and biological research. Aggression and Violent Behavior, 16, 512-524.

Reingle, J. M., Jennings, W. G., Connell, N. M., Businelle, M. S., \& Chartier, K. (2014). On the pervasiveness of event-specific alcohol use, general substance use, and mental health problems as risk factors for intimate partner violence. Journal of Interpersonal Violence, 29, 2951-2970.

Retz, W., Retz-Junginger, P., Supprian, T., Thome, J., \& Rosler, M. (2004). Association of serotonin transporter promoter gene polymorphism with violence: relation with personality disorders, impulsivity, and childhood ADHD psychopathology. Behavioral Sciences \& the Law, 22, 415-425.

Roberts, A. L., McLaughlin, K. A., Conron, K. J., \& Koenen, K. C. (2011). Adulthood stressors, history of childhood adversity, and risk of perpetration of intimate partner violence. American Journal of Preventive Medicine, 40, 128-138.

Rossell, D. R.,\& Siever, L. J. (2015). The neurobiology of aggression and violence. CNS Spectrums, 20, 254-279.

Sabol, S. Z., Hu, S., \& Hamer, D. (1998). A functional polymorphism in the monoamine oxidase a gene promoter. Human Genetics, 103, 273-279.

Saunders, J. B., Aasland, O. G., Babor, T. F., De La Fuente, J. R., \& Grant, M. (1993). Development of the alcohol use disorders identification test (AUDIT): WHO collaborative project on early detection of persons with harmful alcohol consumption-II. Addiction, 88, 791-804.

Schwandt, M. L., Lindell, S. G., Sjöberg, R. L., Chisholm, K. L., Higley, J. D., Suomi, S. J., et al. (2010). Gene-environment interactions and response to social intrusion in male and female rhesus macaques. Biological Psychiatry, 64, 323-330.

Stein, M. B., Schork, N. J., \& Gelernter, J. (2008). Gene-by-environment (serotonin transporter and childhood maltreatment) interaction for anxiety sensitivity, and intermediate phenotype for anxiety disorders. Neuropsychopharmacology, 33, 312-319.

Straus, M. A. (2004). Prevalence of violence against dating partners by male and female university students worldwide. Violence Against Women, 10, 790-811.

Straus, M. A., Hamby, S. L., Boney-McCoy, S., \& Sugarman, D. B. (1996). The revised conflict tactics scales (CTS2): development and preliminary psychometric data. Journal of Family Issues, 17, 283-316.

Stuart, G. L., McGeary, J. E., Shorey, R. C., \& Knopik, V. S. (2014a). Further investigation of genetics and intimate partner violence. Violence Against Women, 20, 420-426.

Stuart, G. L., McGeary, J. E., Shorey, R. C., Knopik, V. S., Beaucage, K., \& Temple, J. R. (2014b). Genetic associations with intimate partner violence in a sample of hazardous drinking men in batterer intervention programs. Violence Against Women, 20, 385-400.

Taylor, S. (1967). Aggressive behavior and physiological arousal as a function of provocation and the tendency to inhibit aggression. Journal of Personality, 35, 297-310. 
Tikkanen, R., Sjöberg, R. L., Ducci, F., Goldman, D., Holi, M., Tiihonen, J., \& Virkkunen, M. (2009). Effects of MAOA-genotype, alcohol consumption, and aging on violent behavior. Alcoholism, Clinical and Experimental Research, 33, 428-434.

Turkheimer, E., Haley, A., Waldron, M., D’Onofrio, B., \& Gottesman, I. I. (2003). Socioeconomic status modifies heritability of IQ in young children. Psychological Science, 14, 623-628.

Tuvblad, C., Raine, A., Zheng, M., \& Baker, L. A. (2009). Genetic and environmental stability differs in reactive and proactive aggression. Aggressive Behavior, 35, 437-452.

Verhoeven, F. E. A., Booij, L., Kruijt, A.-W., Cerit, H., Antypa, N., \&Van der Does, W. (2012). The effects of $M A O A$ genotype, childhood trauma, and sex on trait and state-dependent aggression. Brain and Behavior: A Cognitive Neuroscience Perspective, 2, 806-813.

Waltes, R., Chiocchetti, A. G., \& Freitag, C. M. (2015). The neurobiological basis of human aggression: a review on genetic and epigenetic mechanisms. American Journal of Medical Genetics Part B, 171B, 650-675.

Watkins, L. E., DiLillo, D., \& Maldonado, R. C. (2015). The interactive effects of emotion regulation strategies and alcohol intoxication on intimate partner aggression. Psychology of Addictive Behaviors, 29, 653-663.

Wendland, J., Martin, B., Kruse, M., Lesch, K.-P., \& Murphy, D. (2006). Simultaneous genotyping of four functional loci of human SLC6A4, with a reappraisal of 5-HTTLPR and rs25531. Molecular Psychiatry, 11, 224-226.

Weyler, W., Hsu, Y.-P. P., \& Breakefield, X. O. (1990). Biochemistry and genetics of monoamine oxidase. Pharmacology \& Therapeutics, 47, 391-417.

Wilkowski, B. M., Robinson, M. D., \& Troop-Gordon, W. (2010). How does cognitive control reduce anger and aggression? The role of conflict monitoring and forgiveness processes. Journal of Personality and Social Psychology, 98, 830-840.

Wright, K. D., Asmundson, G. J., McCreary, D. R., Scher, C., Hami, S., \& Stein, M. B. (2001). Factor validity of the childhood trauma questionnaire in men and women. Depression and Anxiety, 13, 179-183. 